\title{
Investigation of dark counts in innovative materials for superconducting nanowire single- photon detector applications
}

L. Parlato, M. Ejrnaes, U. Nasti, R. Arpaia, T. Taino, et al.

L. Parlato, M. Ejrnaes, U. Nasti, R. Arpaia, T. Taino, T. Bauch, H. Myoren, Roman Sobolewski, F. Tafuri, F. Lombardi, R. Cristiano, G. Pepe, "Investigation of dark counts in innovative materials for superconducting nanowire single-photon detector applications," Proc. SPIE 10229, Photon Counting Applications 2017, 102290 I (15 May 2017); doi: 10.1117/12.2267647

SPIE. Event: SPIE Optics + Optoelectronics, 2017, Prague, Czech Republic 


\title{
Investigation of dark counts in innovative materials for superconducting nanostripe single-photon detector applications
}

\author{
L. Parlato,,${ }^{1,2^{*}}$ M. Ejrnaes,${ }^{2}$ U. Nasti, ${ }^{3}$ R. Arpaia,${ }^{4}$ T. Taino, ${ }^{4}$ T. Bauch, ${ }^{4}$ H. Myoren, ${ }^{5}$ Roman \\ Sobolewski ${ }^{6}{ }^{6}$ F. Tafuri,,${ }^{1,2}$, F. Lombardi, ${ }^{4}$ R. Cristiano, ${ }^{2}$ and G. Pepe ${ }^{1,2}$ \\ ${ }^{1}$ Dipartimento di Fisica, Università degli Studi di Napoli Federico II, I-80125 Napoli, Italy \\ ${ }^{2}$ CNR-SPIN Institute of Superconductors, Innovative Materials and Devices, I-80125 Napoli, Italy \\ ${ }^{33}$ School of Engineering Electronic, University of Glasgow, Glasgow, UK \\ ${ }^{4}$ Quantum Device Physics Laboratory, Department of Microtechnology and Nanoscience, \\ Chalmers University of Technology, S-41296 Göteborg, Sweden \\ ${ }^{5}$ Graduate School of Science and Engineering, Saitama University, 338-8570 Saitama, Japan \\ ${ }^{6}$ Department of Electrical and Computer Engineering, Department of Physics and Astronomy, and \\ Laboratory for Laser Energetics, University of Rochester, New York 14627-0231, USA
}

\begin{abstract}
The phenomenon of dark counts in nanostripes of different superconductor systems such as high-temperature superconducting $\mathrm{YBa}_{2} \mathrm{Cu}_{3} \mathrm{O}_{7-\mathrm{x}}$ and superconductor/ferromagnet hybrids consisting of either $\mathrm{NbN} / \mathrm{NiCu}$ or $\mathrm{YBa}_{2} \mathrm{Cu}_{3} \mathrm{O}_{7}$ ${ }_{x} / \mathrm{L}_{0.7} \mathrm{Sr}_{0.3} \mathrm{MnO}_{3}$ bilayers have been investigated. For $\mathrm{NbN} / \mathrm{NiCu}$ the rate of dark-count transients have been reduced with respect to pure $\mathrm{NbN}$ nanostripes and the events were dominated by a single vortex entry from the edge of the stripe. In the case of nanostripes based on $\mathrm{YBa}_{2} \mathrm{Cu}_{3} \mathrm{O}_{7-\mathrm{x}}$, we have found that thermal activation of vortices was also, apparently, responsible for triggering dark-count signals.
\end{abstract}

Keywords: Single-photon detectors, superconducting photon detectors, hybrid materials, high-critical temperature superconductors, dark counts.

\section{INTRODUCTION}

Superconducting nanostripe single-photon detectors (SNSPDs) [1] are currently object of great interest in the field of quantum optics, quantum key distribution, and space telecommunications, because of their ultrahigh counting rate (subgigahertz), ultralow jitter ( $<30 \mathrm{ps}$ ), very high detection efficiency (theoretically close to $100 \%$ ) for wavelengths up to the near infrared, very low dark counts $(<1 \mathrm{~Hz}$ ), and ultrafast time response (few ps) [2]. The best realizations of these devices in terms of their counting performance, feasibility of fabrication, and working temperature, are presently based on ultrathin layers of $\mathrm{NbN}[3,4]$, although alternative materials have been proposed with the aim of improving the SNSPD efficiency and signal-to-noise ratio, in particular, at longer infrared wavelengths. Some examples are transition metal silicides, like WSi [5-7] and MoSi [8] or MoGe [9].

Typically, SNSPDs consist of about 200-nm-wide and about 4-nm-thick superconducting nanostripes forming an extensive (up to $10 \times 10 \mu \mathrm{m}^{2}$ ) meander structure. The operation principle of an SNSPD is based on a current-assisted transition from the superconducting state to the resistive regime induced by absorption of a photon. The superconducting nanostripe operates at a temperature far below its critical temperature $T_{\mathrm{C}}$ and is biased sufficiently close to its critical current $I_{\mathrm{C}}$, so even absorbed energy of a single optical photon is sufficient to generate a large enough hotspot, i.e., superconductivity-suppressed local region in the stripe, to trigger a local transition to the resistive state, resulting in a voltage pulse, recorded as a photon detection event.

Independently of photon events, in a current-biased SNSPD, one can observe spontaneous transient voltage pulses generated without photon absorption and typically known as dark-count events. Dark counts mimic photon counts and, obviously, adversely affect the detector performance. The origin of dark counts in SNSPDs, recently, attracted a special

Photon Counting Applications 2017, edited by Ivan Prochazka, Roman Sobolewski, Ralph B. James, Proc. of SPIE Vol. 10229, 102290I · @ 2017 SPIE · CCC code: 0277-786X/17/\$18 · doi: 10.1117/12.2267647 
attention in literature and much research has been devoted to the physical origin of these unwanted events, focusing on revealing various mechanisms that, generally, involve thermal activation of magnetic vortices in 2-dimensional (2D) superconducting stripes [10-12]. Another thrust is aiming to develop novel materials systems that enable, hopefully substantial, reduction of dark counts. Within this idea, we have investigated the phenomenon of dark counts in nanostripes based on high- $T_{\mathrm{C}}$ superconducting $\mathrm{YBa}_{2} \mathrm{Cu}_{3} \mathrm{O}_{7-\mathrm{x}}(\mathrm{YBCO})$ and superconductor/ferromagnet $\mathrm{NbN} / \mathrm{NiCu}$ and $\mathrm{YBa}_{2} \mathrm{Cu}_{3} \mathrm{O}_{7-\mathrm{x}} / \mathrm{L}_{0.7} \mathrm{Sr}_{0.3} \mathrm{MnO}_{3}$ (YBCO/LSMO) hybrids. In the case of hybrid structures, our previous studies have shown that the presence of an ultrathin ferromagnetic overlayer influences the superconducting properties of the bilayer, in terms of the critical current density $J_{\mathrm{C}}, T_{\mathrm{C}}$, as well as the electron relaxation time [13-16]. In work presented here, we focus on dark-count measurements in hybrid, superconductor/ferromagnet $(\mathrm{S} / \mathrm{F})$ stripes patterned from nanobilayers consisting of ultrathin $\mathrm{NiCu}$ weak ferromagnetic films deposited top of ultrathin $\mathrm{NbN}$ films, as well as from pure $\mathrm{NbN}$ films fabricated in the same conditions and treated as a reference. We stress that our work shows that $\mathrm{NbN} / \mathrm{NiCu}$ nanostripes exhibit a drastic decrease of dark-count rates, as compared to pure $\mathrm{NbN}$ specimens, especially in the near- $I_{\mathrm{C}}$ bias region, where the SNSPD has the highest quantum efficiency.

Independently, recent, extensive efforts have been made to explore feasibility of SNSPDs based on YBCO [17-21]. The YBCO obvious advantage is its very high $T_{\mathrm{C}}$, intrinsic, a few-ps-long quasiparticle recombination time [15, 21], as well as other desired features of an unconventional superconducting material [22]. On the other hand, YBCO exhibits a coherence length of about $2 \mathrm{~nm}$ [23], making it extremely difficult to fabricate ultrathin and ultra-narrow superconducting nanostripes needed for effective photon detection. Here, we relate observation of dark counts in 10-nmthick YBCO nanostripes to the mechanisms observed in NbN-based SNSPDs. We also investigate the prominent role of a ferromagnetic overlayer LSMO, epitaxially grown on top the YBCO film. LSMO is both chemically and structurally compatible with YBCO and its layer acts as a capping/protection for YBCO, minimizing degradation of its superconducting properties, especially when an ultrathin base film is fabricated. Simultaneously, LSMO is magnetic, so studies of dark-count events in LSMO/YBCO nano-layers are very important in the development of the YBCO-based SNSPDs. Thus, our work moves us a step forward to the demonstration that YBCO nanostripes can indeed work as efficient SNSPDs at much higher temperatures.

\section{EXPERIMENTAL DETAILS}

\subsection{Fabrication}

Briefly, 8-nm-thick $\mathrm{NbN}$ films have been deposited on $\mathrm{MgO}$ substrates by reactive dc-magnetron sputtering in an $\mathrm{Ar} / \mathrm{N}_{2}$ gas mixture at a power of $360 \mathrm{~W}$. 6-nm NiCu overlayer has been next deposited without breaking the vacuum by dc magnetron sputtering from a $\mathrm{Ni}_{0.39} \mathrm{Cu}_{0.61}$ target at a power of $155 \mathrm{~W}$ and a deposition rate of $60 \mathrm{~nm} / \mathrm{min}$. Magnetic susceptibility tests demonstrated that our $\mathrm{NiCu}$ overlayers were ferromagnetic with the Curie temperature of about $20 \mathrm{~K}$. Gold contact pads have been prepared by conventional photolithography and lift-off method, while nanostripes have been patterned by electron beam lithography, followed by a reactive-ion-etching method. For thermal fluctuation measurements, we used 5- $\mu \mathrm{m}$-long, straight nanostripes with the widths of $175 \mathrm{~nm}$ and $100 \mathrm{~nm}$, for $\mathrm{NbN}$ and $\mathrm{NbN} / \mathrm{NiCu}$, respectively.

For YBCO-based experiments, 10-nm-thick films of YBCO have been deposited on heated MgO (110) substrates by pulsed laser deposition (PLD). In addition, a carbon hard mask has been deposited by PLD at room temperature, acting as a protection layer for YBCO during its patterning, i.e., to avoid a direct impact of $\mathrm{Ar}$ ions during the etching process. The details of the process can be found in [24]. In the case of oxide S/F bilayers, on the top of a 10-nm YBCO film, an extra 15-nm-thick top, LSMO layer has been deposited ex situ by PLD [18]. Next, the bilayers were patterned to form short, 65-nm- and 100-nm-wide nanostripes.

\subsection{Experimental results: $\mathrm{NbN} / \mathrm{NiCu}$ nanostripes}

In order to investigate the transport properties and dark-count rates, our samples were mounted on a cryogenic insert that was, subsequently, placed inside a standard, helium transport Dewar. The sample holder was surrounded by a metallic enclosure, completely shielding the test structure from outside radiation. The sample temperature was controlled by 
varying the helium vapor pressure and position of the insert inside the Dewar, and was measured with a calibrated germanium thermometer. $\mathrm{NbN}$ stripes showed critical temperature $T_{\mathrm{C}}=12.1 \pm 0.2 \mathrm{~K}$, and critical current density $J_{\mathrm{C}}{ }^{\mathrm{NbN}}=$ 11.6 MA $/ \mathrm{cm}^{2}$ at $T=4.2 \mathrm{~K}$. The $T_{\mathrm{C}}$ values of our $\mathrm{NbN} / \mathrm{NiCu}$ samples were lower by less than $0.5 \mathrm{~K}$, while their $J_{\mathrm{C}}$ values, also measured at $T=4.2 \mathrm{~K}$, were clearly enhanced and equal to $J_{\mathrm{C}}^{\mathrm{NbN} / \mathrm{NiCu}}=43.2 \mathrm{MA} / \mathrm{cm}^{2}$. The $J_{\mathrm{C}}$ enhancement in S/F nanobilayers has been observed earlier [13] and can be related to the magnetic overlayer, considered as scalar impurities that generate extra flux pinning [25].

The dark count events were registered as voltage fluctuation transients and read out using a cascade of two microwave amplifiers with an effective bandwidth of $0.1-100 \mathrm{MHz}$ and a total gain of $20 \mathrm{~dB}$. The amplified signals were fed with a $50-\Omega$ coaxial cable into our readout electronics that consisted of either a digital oscilloscope with a 1-GHz bandwidth or a pulse counter with a $100-\mathrm{MHz}$ bandwidth. Figure 1 shows dark-count pulses observed on the oscilloscope for $\mathrm{NbN}(8$ $\mathrm{nm})$ and $\mathrm{NbN}(8 \mathrm{~nm}) / \mathrm{NiCu}(6 \mathrm{~nm})$ nanostripes, respectively, recorded under the same bias conditions, namely, $I_{\mathrm{b}} / I_{\mathrm{C}}=0.8$ and $T=5 \mathrm{~K}$. The shapes of both pulses are identical, but, clearly, the amplitude of the $\mathrm{NbN} / \mathrm{NiCu}$ pulse is significantly larger, in full agreement with the $J_{\mathrm{C}}$ enhancement for the $\mathrm{NbN} / \mathrm{NiCu}$ nanostripes as shown in the inset in Fig. 1.

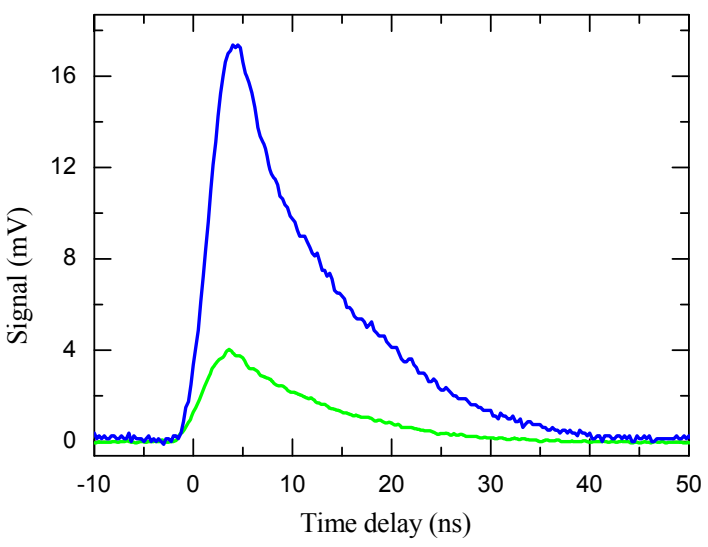

Figure 1. Time-resolved dark-count signal recorded for $\mathrm{NbN}(8 \mathrm{~nm})$ (green line) and $\mathrm{NbN}(8 \mathrm{~nm}) / \mathrm{NiCu}(6 \mathrm{~nm})($ blue line) nanostripes at $I_{\mathrm{b}} / I_{\mathrm{C}}=0.8$ and $T=5 \mathrm{~K}$. The inset presents the corresponding current-voltage $(I-V)$ characteristics of $\mathrm{NbN}(8 \mathrm{~nm})(\mathrm{green}$ line $)$ and $\mathrm{NbN}(8 \mathrm{~nm}) / \mathrm{NiCu}(6 \mathrm{~nm})$ (red line) samples.
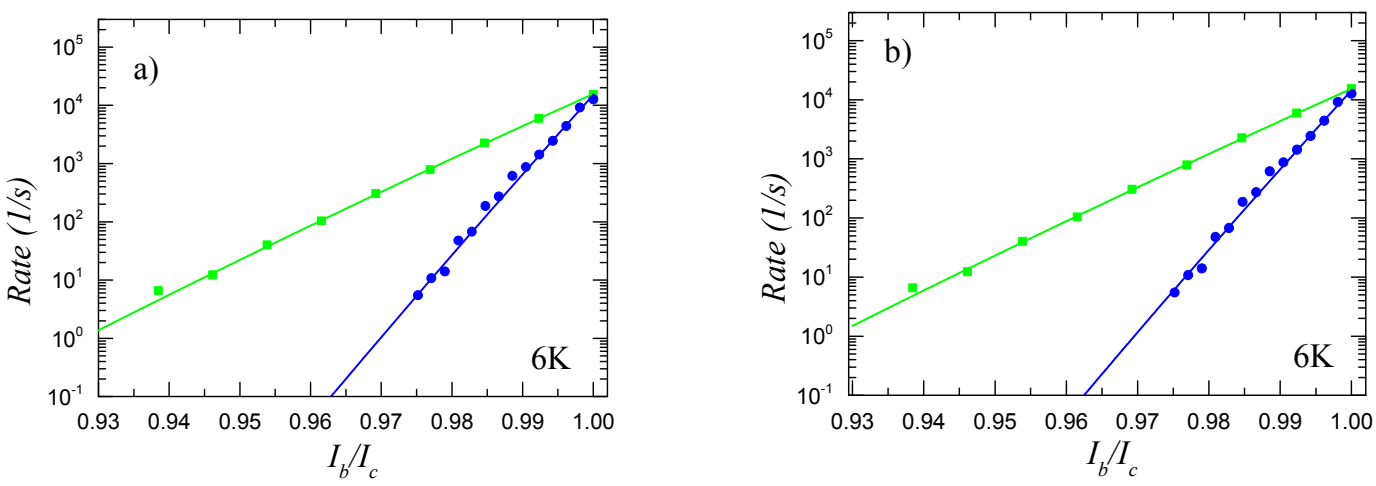

Figure 2. Dark-count rates versus the normalized bias current measured at $6 \mathrm{~K}$, for a pure $\mathrm{NbN}(8 \mathrm{~nm})$ nanostripe (black squares) and for $\mathrm{NbN}(8 \mathrm{~nm}) / \mathrm{NiCu}(6 \mathrm{~nm}) \mathrm{S} / \mathrm{F}$ nanostructures (red circles). The solid lines are the best fits obtained using the VAP model (a) and the VH model (b), respectively. 
Despite of their enhanced amplitudes, the recorded rates of dark counts for our $\mathrm{S} / \mathrm{F}$ test samples were found to be much lower than those observed in the pure $\mathrm{NbN}$ reference sample, as seen in Fig. 2. The latter can be viewed as a clear advantage of the hybrid S/F structures. The investigation of the physical origin of dark counts in S/F nanostructures have been reported by us in [26]. Our experimental results can be understood in terms of either the model based on thermal activation of vortex-antivortex pairs (VAPs) or the one based on thermal hopping of single unbounded vortices (VHs) $[10,11]$. In both cases, motion of VAPs or VHs creates a normal-conducting, resistive domain across a 2D nanostripe and results in a voltage transient that is registered as a dark-count event. Both vortex-based models can explain the significant decrease of dark counts in S/F nanostripes, observed in Fig. 2, since the presence of the F layer leads in a stronger vortex pinning in the $\mathrm{S}$ layer that, in turn, is responsible for both the $J_{\mathrm{C}}$ enhancement and reduction of the rate of dark-counts events. However, a comparison between the temperature dependence of the vortex-generation energy barrier in the case of both models indicates that the VH scenario better explains the behavior for S/F nanostripes, whereas the VAP scenario is preferred to explain the dark-count mechanism in pure $\mathrm{S}$ nanostripes, such as $\mathrm{NbN}$ [26].

\subsection{Experimental results: $\mathrm{YBCO}$ and $\mathrm{YBCO} / \mathrm{LSMO}$ nanostripes}

Both pure YBCO and YBCO/LSMO bilayer nanostripes have been characterized by measuring their both resistivity vs. temperature $R(T)$ and $I-V$ characteristics in the whole temperature range from room temperature down to $T=5 \mathrm{~K}$. For a $\mathrm{YBCO} / \mathrm{LSMO}$ nanostripe, $T_{\mathrm{C}}=82 \pm 6 \mathrm{~K}$ was determined, and a relatively broad superconducting transition was, apparently, due to a stripe superconducting properties degradation during the nano-bilayer patterning. The maximum $J_{\mathrm{C}}$ value was found to be as large as $20 \mathrm{MA} / \mathrm{cm}^{2}$. In 50-nm-thick, pure YBCO devices, the $I-V$ curves exhibited a gradual superconducting-to-resistive transition with a well-established flux-flow regime, where a resistive state locally appears, but the superconductivity is still present in the nanostripe [19]. Therefore, the photo-response behavior was markedly different from that observed in NbN-based SNSPDs, and the main contribution to the photo-response had a bolometric nature.
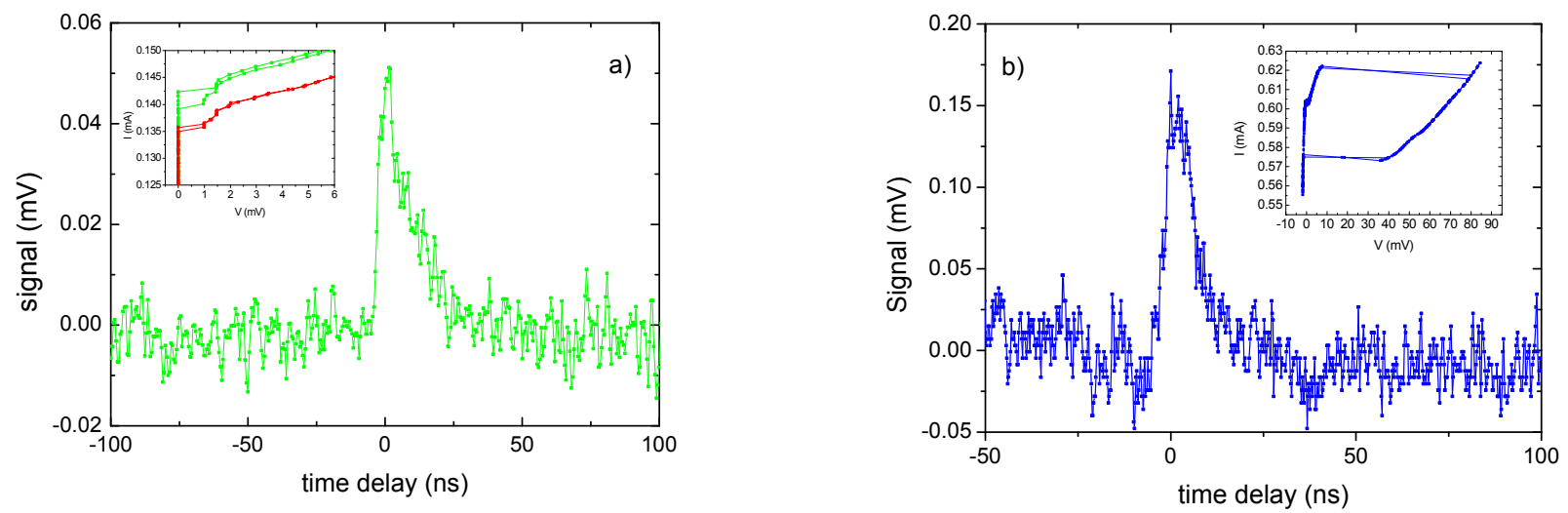

Figure 3. Time-domain, dark-count voltage signals for (a) YBCO and (b) YBCO/LSMO nanostripes, respectively, recorded for $I_{\mathrm{b}} / I_{\mathrm{C}}=$ 0.9 and at $T=5 \mathrm{~K}$. In the insert current-voltage characteristics of YBCO nanostripe at $T=5 \mathrm{~K}$ (green line) and $T=10 \mathrm{~K}$ (red line) and $\mathrm{YBCO} / \mathrm{LSMO}$ at $T=5 \mathrm{~K}$ (blue line).

Figures 3(a) and 3(b) show time-domain dark-count voltage pulses for a $10 \mathrm{~nm}$-thick and 65 -nm-wide YBCO and YBCO/LSMO nanostripes, respectively, measured for $I_{\mathrm{b}} / I_{\mathrm{C}}=0.9$ and $T=5 \mathrm{~K}$. Measurements were performed at the experimental setup the same as one used for testing metallic nanostripes. Both waveforms presented in Fig. 4, actually, have the identical shape with the detection-system-limited rise time and about a 30-ns-long fall time. The inset in each panel shows the corresponding $I-V$ curve and in the case of Fig. 4(a) we observe a sharp, of the order of a few $\mathrm{mV}$, voltage switch (black dots, $T=5 \mathrm{~K}$ and red dots, $T=10 \mathrm{~K}$ ), as the bias crosses $I_{\mathrm{C}}$, and a small hysteresis. The hysteresis decreases with the increase of temperature (see red dots), disappearing at temperatures higher than 15-20 K. The presence of a voltage switch and of a hysteresis can be related to a motion of the vortex lattice and/or of Joule heating of the sample. In the case of YBCO/LSMO nanostripes, we observe an increase of $I_{\mathrm{C}}$ [see inset in Fig. 3(b)] as expected in 
a S/F bilayer, as well as the substantial increase of the hysteresis. The amplitude of the dark count is also correspondingly larger, since in both cases the test was performed at the same normalized bias current $\left(I_{\mathrm{b}} / I_{\mathrm{C}}=0.9\right)$. Direct observation of dark transients in our both YBCO and YBCO/LSMO nanostripes is an important step in the development of YBCO-based SNSPDs.

\section{CONCLUSIONS}

We report on our progress in understanding of the physical origin of dark-count transients spontaneously generated in current-biased SNSPDs, focusing on S/F-type nanostripes. We demonstrate that the presence of an ultrathin F layer, not only enhances the maximum value of $J_{\mathrm{C}}$, but in $\mathrm{NbN} / \mathrm{NiCu}$ nanostructures significantly reduces the rate of appearance of dark-count transients. This behavior can be related to thermally-activated single vortex or vortex-antivortex pairs that move across a 2D superconducting stripe. We have also observed dark-count events in both YBCO and YBCO/LSMO nanostripes, in a similar way to those observed in our metallic SNSPD nanostructures. Dart-count transients appeared at low temperatures, when the corresponding $I-V$ characteristics exhibited a hysteretic behavior. The latter can be explained by a dissipation mechanism due to the motion of vortices in the nanostripes. The use of an ultrathin LSMO cap layer did not affect the superconducting transport properties of the YBCO, but allowed us to minimize material degradation during the nanostripe patterning. Interestingly, magnetic property of the LSMO layer led to an enhancement of the S/F nanostripe $I_{\mathrm{C}}$, apparently, in a similar way as it has been observed on all-metallic S/F bilayers [13]. The latter result can be regarded as a first step towards implementation HTS materials in SNSPDs. Thus, we can conclude that the use of S/F nanostructures has a beneficial effect on photon-counting performance of SNSPDs based on both low- and high- $T_{\mathrm{C}}$ superconductors.

\section{ACKNOWLEDGEMENT}

Research in Rochester has been supported in part by the grant from the HYPRES Co., and by the New York State Advanced Technology Centers for Innovative and Enabling Technologies (University of Rochester) and Advanced Sensor Technologies (Stony Brook University).

\section{REFERENCES}

[1] Gol'tsman, G. N., Okunev, O., Chulkova, G., Lipatov, A., Semenov, A., Smirnov, K., Voronov, B., Dzardanov, A., Williams, C., Sobolewski, R., "Picosecond superconducting single-photon optical detector" Appl. Phys. Lett. 79, 705-707 (2001)

[2] Natarajan, C. M., Tanner, M. G and Hadfield, R. H. "Superconducting nanowire single-photon detectors: physics and applications" Supercond. Sci. Technol. 25, 063001 and references therein (2012).

[3] Lusche R., Semenov A., Ilin K., Siegel M., Korneeva Y., Trifonov A., Korneev A., Goltsman G., Vodolazov D., and Hübers H. W., "Effect of the wire width on the intrinsic detection efficiency of superconducting-nanowire singlephoton detectors" J. of Appl. Phys. 116, 043906 (2014)

[4] Ilin K., Henrich D., Luck Y., Liang Y., Siegel M., and Vodolazov D. Yu., "Critical current of Nb, NbN, and TaN thin-film bridges with and without geometrical nonuniformities in a magnetic field" Phys. Rev. B 89, 184511 (2014)

[5] Korneeva Y.P., Mikhailov M Yu, Pershin Yu P, Manova N N, Divochiy A V, Vakhtomin Yu B, Korneev A A, K V Smirnov, Sivakov A G, Devizenko A Yu, "Superconducting single-photon detector made of MoSi film" Supercond. Sci. Technol. 27, 095012 (2014)

[6] Verma V. B., Korzh B., Bussières F., Horansky R. D., Lita A. E., Marsili F., Shaw M. D., Zbinden H., Mirin R. P., and Nam S. W., "High-efficiency WSi superconducting nanowire single-photon detectors operating at $2.5 \mathrm{~K}$ " Appl. Phys. Lett 105, 122601 (2014);

[7] Marsili F., Stevens M. J., Kozorezov A., Verma V. B., Lambert C., Stern J. A., Horansky R. D, Dyer S., Duff S., Pappas D.P., Lita A. E., Shaw M. D., Mirin R. P., and Nam S. W., "Hotspot Relaxation Dynamics in a Current Carrying Superconductor" Phys. Rev. B 93, 094518 (2016); 
[8] Zhang X., Engel A., Wang Q., Schilling A., Semenov A., Sidorova M., Hübers H.-W., Charaev I., Ilin K., and Siegel M., "Characteristics of superconducting tungsten silicide WSi for single photon detection" Phys. Rev B 94, 174509 (2016)

[9] Verma V.B., Lita A.E., Vissers M.R., Marsili F., Pappas D. P., Mirin R.P., Nam S.W. "Superconducting nanowire single photon detectors fabricated from an amorphous Mo0.75Ge0.25 thin film" Appl. Phys. Let. 105. 022602, (2014)

[10] Bartlof, H., Engel, A., Schilling, A., Il'in, K., Siegel, M., Huebers, H.W., Semenov, A., "Current Assisted, Thermally Activated Flux Liberation in Ultrathin Nanopatterned NbN Superconducting Meander Structures" Phys. Rev. B 81, 024502-1_12 (2010)

[11] Bulaevskii, L.N., Graf, M.J., Batista, C.D., Kogan, V.G., "Vortex-induced dissipation in narrow current-biased thinfilm superconducting strips" Phys. Rev. B 83, 144526-1_9 (2011)

[12] Engel. A., Renema J.J., Ill'in K. and Semenov A., “ Detection mechanism of superconducting nanowire singlephoton detectors" Supercond. Sci. Technol. 28114003 (2015)

[13] Marrocco, N., Pepe, G.P., Capretti, A., Parlato, L., Pagliarulo, V., Peluso, G., Barone, A., Cristiano, R., Ejrnaes, M., Casaburi, A., Kashiwazaki, N., Taino, T., Myoren, H., and Sobolewski R., "Strong critical current density enhancement in $\mathrm{NiCu} / \mathrm{NbN}$ superconducting nanostripes for optical detection" App. Phys. Lett. 97, 092504-1_3 (2010)

[14] Pan, D., Pepe, G.P., Pagliarulo, V., De Lisio, C., Parlato, L., Khafizov, M., Komissarov, I., and Sobolewski R., "Layered ferromagnet/superconductor heterostructures: Nonequilibrium quasiparticle dynamics and photodetector applications" Phys. Rev. B 78, 174503-1_7 (2008)

[15] Parlato, L., Arpaia, R., De Lisio, C. , Miletto Granozio, F., Pepe, G. P., Perna, P., Pagliarulo, V., Bonavolontà, C., Radovic, M. , Wang, Y., Sobolewski, R., and Scotti di Uccio, U., "Time-resolved optical response of all-oxide YBa2Cu3O7/La0.7Sr0.3MnO3 proximitized bilayers" Phys. Rev B 87, 134514 (2013)

[16] Cirillo, C.; Pagliarulo, V; Myoren, H; Bonavolonta, C; Parlato, L; Pepe, GP; Attanasio, C , "Quasiparticle energy relaxation times in $\mathrm{NbN} / \mathrm{CuNi}$ nanostripes from critical velocity measurements" Phys. Rev. B vol. 84, 054536 (2011)

[17] Curtz N., Koller E, Zbinden H, Decroux M, Antognazza L, Fischer Ø and Gisin N, "Patterning of ultrathin YBCO nanowires using a new focused-ion-beam process" Supercond. Sci. Technol. 23, 045015 (2010)

[18] Arpaia R, Ejrnaes M, Parlato L, Cristiano R, Arzeo M, Bauch T, Nawaz S, Tafuri F, Pepe G P, Lombardi F., "Highly homogeneous YBCO/LSMO nanowires for photoresponse experiments" Supercond. Sci. Technol. 27, 044027 (2014)

[19] Arpaia R, Ejrnaes M, Parlato L, Tafuri F., Cristiano R, Golubev D., Sobolewski R., Bauch T., Lombardi F., Pepe G.P., "High-temperature superconducting nanowires for photon detection" Physica C 509, 16 (2015)

[20] Lyatti M., Savenko A., and Poppe U., "Ultra-thin YBa2Cu3O7-x films with high critical current density" Supercond. Sci. Technol. 29, 065017 (2016)

[21] Pepe G.P., Parlato L., Marrocco N., Pagliarulo V., Peluso G., Barone A., Tafuri F., Scotti di Uccio U., Miletto F., Radovic M., Pan D., Sobolewski R., "Novel superconducting proximized heterostructures for ultrafast photodetection" Cryogenics 49, 660 (2009)

[22] Bonavolontà C., Parlato L, Pepe G P, de Lisio C., Valentino M., Bellingeri E., Pallecchi I., Putti M. and Ferdeghini C., "Ultrafast quasiparticle relaxation dynamics in high quality epitaxial FeSe0.5Te0.5 thin films Supercond." Sci. Technol. 26, 075018 (2013)

[23] Papari G., Carillo F, Stornaiuolo D, Longobardi L, Beltram F and Tafuri F., "High critical current density and scaling of phase-slip processes in YBaCuO nanowires" Supercond. Sci. Technol. 25, 035011 (2012)

[24] R. Arpaia, et al. submitted to Physical Review Applied (2017).

[25] A.I. Buzdin, "Proximity effects in superconductor-ferromagnet heterostructures" Rev. Mod. Phys. 77, 935 (2005).

[26] Nasti, U., Parlato, L., Ejrnaes, M., Cristiano, R., Taino, T., Myoren, H., Sobolewski, R., Pepe, G. "Thermal fluctuations in superconductor/ferromagnet nanostripes" Phys. Rev.B 92, 14501 (2015). 